\title{
Intraoperative Assessment of Carotid Endarterectomy Using Near-Infrared Indocyanine Green Video Angiography
}

\author{
Nathan Todnem, Vamsi Reddy , Joseph Withrow and Scott Rahimi
}

Department of Neurosurgery, Augusta University, USA

\begin{abstract}
The benefits and safety of carotid endarterectomy (CEA) for the treatment of carotid artery stenosis are well established. Perioperative complications such as thrombosis, embolism, and subsequent stroke caused by technical error can potentially prevented if detected and addressed while still in the operating room. Intraoperative duplex ultrasonography and arteriography are studies that are frequently employed to evaluate for defects following the procedure. Indocyanine green video angiography (ICGVA) is used frequently by vascular neurosurgeons in other procedures such as aneurysm clipping to directly visualize the vessels and identify potential complications. Recently this technology was employed during three CEA cases for evaluation of the carotid arteries intraoperatively. Good visualization was obtained in all three cases and no post-operative complications were observed. ICGVA represents a safe, feasible, and easy to perform means of detecting operative complications of CEA. This is the first report of this technique being used for carotid endarterectomy in North America.
\end{abstract}

\section{Introduction}

The indications and benefits of CEA in the treatment in carotid artery stenosis have been thoroughly evaluated and documented. In carefully selected patients, surgical intervention is superior to medical therapy for both symptomatic and asymptomatic patients in the prevention of stroke $[1,2]$. The operative benefit of CEA is dependent on the surgeon's ability to perform the procedure with a very low incidence of perioperative complications. Effort is made to minimize postoperative thrombosis and embolism to avoid neurologic complications, such as stroke. Most perioperative neurological events following this procedure are due to thromboembolic events with a significant portion stemming from technical defects, which are largely preventable if detected early and reexplored [3-5]. The technical defects included irregularities in the suture lines, at the proximal or distal extent of the endarterectomy site, or at the site of plication [5]. There is evidence to suggest that otherwise occult technical defects can be detected with intraoperative imaging and effectively corrected to avoid postoperative stroke [6-15]. Indocyanine green video angiography (ICGVA) is an intraoperative imaging technique, which can be used as an alternative to intraoperative duplex and arteriography during CEA.

\section{Methods}

Our protocol is to perform near-infrared indocyanine green video angiography before and after the excision of the carotid incision was repaired and the plaque removal was completed. Patients undergoing a CEA received a standard dose of $25 \mathrm{mg}$ indocyanine green dissolved in 5 $\mathrm{ml}$ of water, which is roughly equal to $0.25 \mathrm{mg}$. Following injection, ICG becomes NIR fluorescent after binding to protein globulins in the blood with only a 10-20 second latency period before visualization is possible. Florescence is induced when the dye comes in contact with the NIR light within the operative field and was recorded with a NIR instrument (infrared 800 module; Carl Zeizz Surgical GmbH, Oberkochen, Germany) integrated in a surgical microscope (OPMI Pentero; Carl Zeiss Surgical $\mathrm{GmbH}$, Oberkochen, Germany).

\section{Results}

The ICA is well visualized and easily interpreted. Below (Figure 1 and Figure 2) is a picture from before and after the removal of the lesions. The technique allows for real time visualization and inspection for flaps. The ad-

*Corresponding author: Vamsi Reddy, Department of Neurosurgery, Augusta University, 1120 15th Street BI3088, Augusta, GA 30912-2900, USA

Accepted: November 14, 2018

Published online: November 16, 2018

Citation: Todnem N, Reddy V, Withrow J, et al. (2018) Intraoperative Assessment of Carotid Endarterectomy Using Near-Infrared Indocyanine Green Video Angiography. J Neurosurg Res Rev 1(1):19-21

Copyright: ( 2018 Todnem N, et al. This is an open-access article distributed under the terms of the Creative Commons Attribution License, which permits unrestricted use, distribution, and reproduction in any medium, provided the original author and source are credited. 
Citation: Todnem N, Reddy V, Withrow J, et al. (2018) Intraoperative Assessment of Carotid Endarterectomy Using Near-Infrared Indocyanine Green Video Angiography. J Neurosurg Res Rev 1(1):19-21
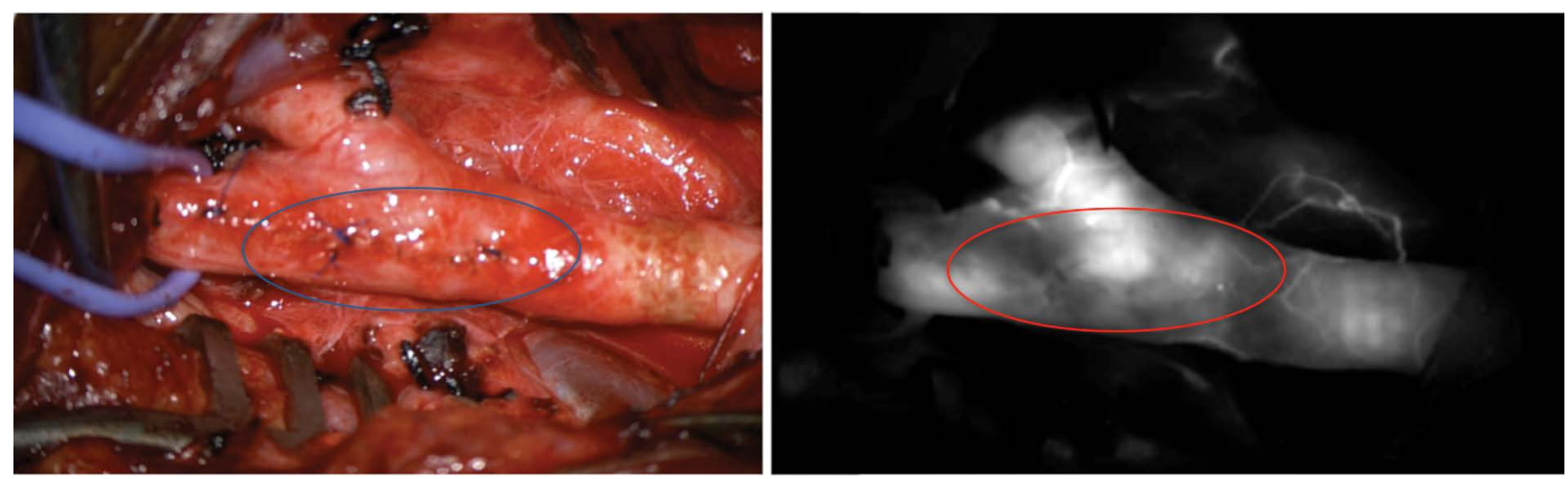

Figure 1: The image on the left shows the real time image following right common carotid endarterectomy compared to the ICG angiography image on the right which shows appropriate filling of the vessel and no intimal tears.
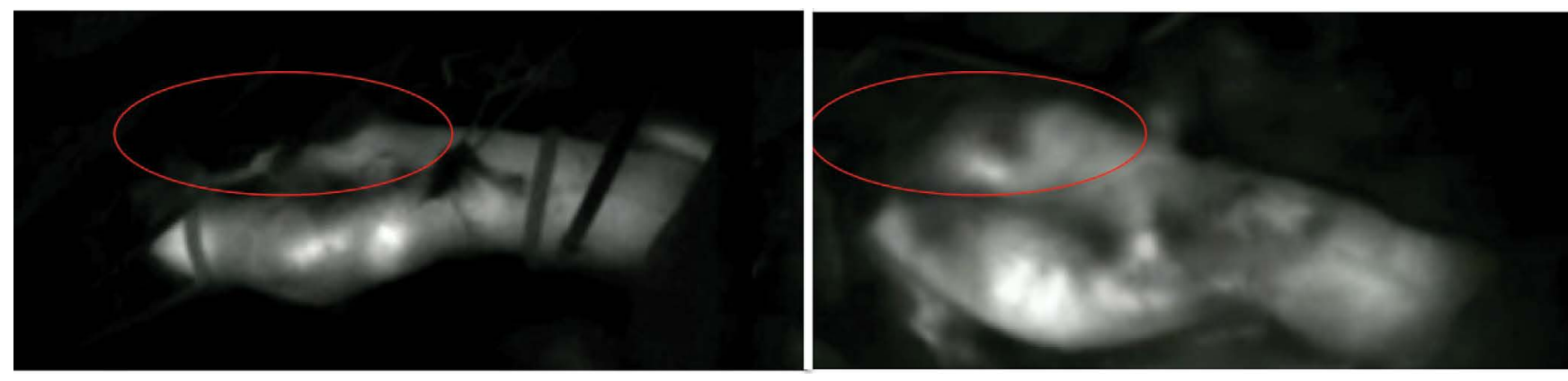

Figure 2: The image on the left shows a lesion and filling defect in the left internal carotid artery. The filling defect is resolved on the image on the right and there is restoration of blood flow in the internal carotid artery.

ditional time added to the procedure is minimal. We are yet to observe any adverse effects from ICG administration. No post-operative strokes have occurred.

\section{Discussion}

Three techniques have classically been the utilized for perioperative evaluation of carotid endarterectomy; continuous wave Doppler, duplex ultrasonography, and intraoperative arteriography. Both intraoperative duplex and arteriography are found to have equivalent sensitivities for identifying major defects, but the sensitivity is believed to be higher for duplex in identifying minor defects ( $87 \%$ vs. $59 \%$ ) [3,8,16-19]. One commonly cited advantage of arteriography is the ability to evaluate ICA distal to the surgical site, however it is unlikely there would be technical defect in this area [20]. Additionally, intraoperative arteriography carries further risk of complications including air emboli, intimal dissection, poor visualization of proximal carotid artery leading to inability to observe flap or clamp injuries in that area, and stroke $[9,16,20,21]$.

A larger study examined the utility of intraoperative carotid Doppler studies and of 650 CEAs, 15 cases required surgical revision for technical defects discovered using intraoperative Doppler [20]. Zero of the 15 patients were reported to have a post-operative stroke [20].
They reported the mean time for duplex scanning was 10 minutes with a standard deviation of 3 minutes, and that they believe duplex to be the gold standard for intraoperative evaluation [20]. However, obtaining the equipment and appropriate training to become proficient in ultrasonography is expensive and unpractical for most neurosurgeons. Adoption of intraoperative ultrasonography likely would require they use an ultrasonography technologist to assist in the study.

Rockman, et al. conducted a literature review that demonstrated the incidence of immediate intraoperative revision based on results from intraoperative imaging to range from $2.3-14.5 \%$ [4]. Addition they also report the incidence of minor defects that were detected but not revised was $20-40 \%$ [4]. Some argue minor defects are a benign finding while others have demonstrated a correlation with minor defects and late restenosis $[4,22]$. The study concluded that routine use of intraoperative imaging studies did not appear to significantly improve perioperative outcomes with regard to ipsilateral perioperative stroke or death [4]. Thus, the value of intraoperative studies remains uncertain although the source suggested selective use of intraoperative imaging techniques when there was a specific concern [4].

While the value remains uncertain, multiple stud- 
Citation: Todnem N, Reddy V, Withrow J, et al. (2018) Intraoperative Assessment of Carotid Endarterectomy Using Near-Infrared Indocyanine Green Video Angiography. J Neurosurg Res Rev 1(1):19-21

ies have demonstrated significant correlation between technical errors in carotid repair and the development of perioperative neurological deficits $[1,2,5,23]$. Despite this fact, most surgeons do not routinely use any type of formal imaging study to identify defects in the arterial repair and rely solely on technique and clinical inspection [4]. ICG video angiography offers a non-invasive method that neurosurgeons are already familiar with to evaluate the repair without significantly lengthening the procedure.

\section{Conclusion}

Intraoperative assessment of carotid endarterectomy using ICGVA could be used to enhance the safety and efficacy of CEA as it has with aneurysm surgery. This real-time technique for intraoperative flow assessment provides an alternative to intraoperative duplex and arteriography. ICGVA offers several advantages to intraoperative duplex and arteriography. There is no obstruction from needle punctures, as experienced in arteriography. No risk of traumatic dissection from contrast injection. No risk of air embolism. No radiation exposure for either the patient or the staff. This technique is already familiar to vascular neurosurgeons who perform aneurysm surgery and can easily be adapted to CEA. ICGVA is safe, easy to perform, interpret, and adds very little additional time to the procedure.

\section{Declarations}

Ethical Approval and Consent to participate.

\section{Competing Interests and Funding}

The authors declare that they have no competing interests and have received no funding for this manuscript.

\section{Author's Contributions}

NT and SR conducted the operations and the collected the data. NT, VR, and JW wrote the initial manuscript and edited the subsequent drafts with help from SR.

\section{References}

1. Barnett HJM, Taylor DW, Haynes RB, et al. (1991) Beneficial effect of carotid endarterectomy in symptomatic patients with high-grade carotid stenosis. N Engl J Med 325: 445-453.

2. (1995) Endarterectomy for asymptomatic carotid artery stenosis. Executive Committee for the Asymptomatic Carotid Atherosclerosis Study. JAMA 273: 1421-1428.

3. Riles TS, Imparato AM, Jacobowitz GR, et al. (1994) The cause of perioperative stroke after carotid endarterectomy. J Vasc Surg 19: 206-216.

4. Rockman CB, Halm EA (2007) Intraoperative imaging: Does it really improve perioperative outcomes of carotid endarterectomy? Semin Vasc Surg 20: 236-243.

5. Rockman CB, Jacobowitz GR, Lamparello PJ, et al. (2000) Immediate reexploration for the perioperative neurologic event after carotid endarterectomy: Is it worthwhile? J Vasc Surg 32: 1062-1070.
6. Bandyk DF, Mills JL, Gahtan V, et al. (1994) Intraoperative duplex scanning of arterial reconstructions: Fate of repaired and unrepaired defects. J Vasc Surg 20: 426-433.

7. Dykes JR 2nd, Bergamini TM, Lipski DA, et al. (1997) Intraoperative duplex scanning reduces both residual stenosis and postoperative morbidity of carotid endarterectomy. Am Surg 63: 50-54.

8. Kinney EV, Seabrook GR, Kinney LY, et al. (1993) The importance of intraoperative detection of residual flow abnormalities after carotid artery endarterectomy. J Vasc Surg 17: 912-923.

9. Mays BW, Towne JB, Seabrook GR, et al. (2000) Intraoperative carotid evaluation. Arch Surg 135: 525-529.

10. Panneton JM, Berger MW, Lewis BD, et al. (2001) Intraoperative duplex ultrasound during carotid endarterectomy. Vasc Surg 35: 1-9.

11. Sawchuk AP, Flanigan DP, Machi J, et al. (1989) The fate of unrepaired minor technical defects detected by intraoperative ultrasonography during carotid endarterectomy. J Vasc Surg 9: 671-676.

12. Schwartz RA, Peterson GJ, Noland KA, et al. (1988) Intraoperative duplex scanning after carotid artery reconstruction: A valuable tool. J Vasc Surg 7: 620-624.

13. Seelig MH, Oldenburg WA, Chowla A, et al. (1999) Use of intraoperative duplex ultrasonography and routine patch angioplasty in patients undergoing carotid endarterectomy. Mayo Clin Proc 74: 870-876.

14. Steinmetz OK, MacKenzie K, Nault P, et al. (1998) Intraoperative duplex scanning for carotid endarterectomy. Eur $\mathrm{J}$ Vasc Endovasc Surg 16: 153-158.

15. Walker RA, Fox AD, Magee TR, et al. (1996) Intraoperative duplex scanning as a means of quality control during carotid endarterectomy. Eur J Vasc Endovasc Surg 11: 364-367.

16. Andersen CA, Collins GJ Jr, Rich NM (1978) Routine operative arteriography during carotid endarterectomy: A reassessment. Surgery 83: 67-71.

17. Lingenfelter KA, Fuller BC, Sullivan TM (1995) Intraoperative assessment of carotid endarterectomy: A comparison of techniques. Ann Vasc Surg 9: 235-240.

18. Towne JB, Weiss DG, Hobson RW $2^{\text {nd }}(1990)$ First phase report of cooperative Veterans Administration asymptomatic carotid stenosis study--operative morbidity and mortality. J Vasc Surg 11: 252-259.

19. Valenti D, Gaggiano A, Berardi G, et al. (2003) Intra-operative assessment of technical defects after carotid endarterectomy: A comparison between angiography and colour duplex scan. Cardiovasc Surg 11: 26-29.

20. Ascher E, Markevich N, Kallakuri S, et al. (2004) Intraoperative carotid artery duplex scanning in a modern series of 650 consecutive primary endarterectomy procedures. J Vasc Surg 39: 416-420.

21. Plecha FR, Pories WJ (1972) Intraoperative angiography in the immediate assessment of arterial reconstruction. Arch Surg 105: 902-907.

22. Baker WH, Koustas G, Burke K, et al. (1994) Intraoperative duplex scanning and late carotid artery stenosis. J Vasc Surg 19: 829-833.

23. DeBakey ME (1975) Successful Carotid Endarterectomy for Cerebrovascular Insufficiency: Nineteen-Year Follow-up. JAMA 233: 1083-1085. 